Somnologie $2016 \cdot 20: 8-15$

DOI 10.1007/s11818-015-0039-z

Eingegangen: 24. Juli 2015

Angenommen: 24. September 2015

Online publiziert: 18. Januar 2016

(c) The Author(s) 2016. This article is available at SpringerLink with Open Access

CrossMark

\author{
Cynthia Marisch ${ }^{1}$ Lisa Genzel ${ }^{2}$ Axel Steiger ${ }^{1} \cdot$ Martin Dresler $^{1,3}$ \\ 'Max-Planck-Institut für Psychiatrie, München, Deutschland \\ ${ }^{2}$ Centre for Cognitive and Neural Systems, University of Edinburgh, Edinburgh, UK \\ ${ }^{3}$ Donders Institute, Radboud University Medical Centre, Nijmegen, Niederlande
}

\title{
Kreativität und Schlaf
}

\section{Kreativität: Konzepte und Modelle}

Hochentwickelte Zivilisationen bauen wesentlich auf innovativen Ideen und Entdeckungen auf. Im Vergleich zu anderen kognitiven Domänen nimmt die systematische Erforschung der Kreativität jedoch noch immer eine Randstellung in der Psychologie und kognitiven Neurowissenschaft ein - daran hat sich auch mehr als ein halbes Jahrhundert nach Guilfords prominenter Klage, Kreativität sei trotz ihrer immensen Bedeutung praktisch unerforscht [43], vergleichsweise wenig geändert. Die thematische Breite und konzeptionelle Offenheit des Kreativitätsbegriffs dürfte dabei eine wesentliche Rolle für die langsame und uneinheitliche Entwicklung der Kreativitätsforschung spielen [25]. Empirienahe Operationalisierungen des Kreativitätsbegriffs umfassen dabei so unterschiedliche Konzepte wie originelle Produktivität, wie sie etwa in Tests divergenten Denkens abgefragt wird, und kreative Problemlösungsfähigkeit, die mit Einsichtsaufgaben zu erfassen versucht wird [1].

Rhodes [77] beschrieb die vier $P$ der Kreativität. Unter dem Oberbegriff der Person fasste er alle Eigenschaften zusammen, die den Charakter eines kreativen Menschen prägen, wie z. B. den Intellekt, das Temperament, Gewohnheiten oder das Selbstbild. Ausgehend hiervon ist dann ein Prozess zur Verarbeitung kreativer Gedanken notwendig. Dieser umfasst unter anderem Motivation, Lernverhalten, Gedankengänge und Kommunikation. Da diese Vorgänge jedoch nicht in völliger Isolation vonstatten gehen, ist auch das Umfeld einer Person äußerst wichtig. Diesen Umstand bringt Rhodes mit dem englischen Wort press zur Geltung, das den Druck der Umwelt auf ein kreatives Individuum, das dadurch geformt wird, zum Ausdruck bringt. Auch Simonton [87] betont diesbezüglich die Vorteile einer herausfordernden Umwelt und kulturellen Diversität für die Entwicklung eines kreativen Geistes. Letztendlich kommt es dann zur Entstehung gewisser Produkte, die sich allerdings nicht auf eine rein materielle Ebene beschränken, sondern auch kreative Gedanken umfassen, die z. B. in Form von Worten oder Kunstwerken Ausdruck finden [77].

Csikszentmihalyi [13, 14] erklärt Kreativität als ein Zusammenspiel von 3 Faktoren, die sich gegenseitig beeinflussten und voneinander abhingen: das Individuum, das Feld und die Domäne. Er betonte, dass ein Individuum nur dann etwas bewegen kann, wenn es genügend Expertise in einem Fachbereich, der Domäne, besitzt. Dabei ist die Interaktion mit Experten aus einem entsprechenden Feld äußerst wichtig, da diese ein entscheidender Maßstab sind, ob die neue Idee von Bestand ist oder nicht. Unterstützung erfährt dieser Ansatz dadurch, dass viele außergewöhnliche Künstler, wie etwa Edvard Munch oder Van Gogh, erst nach langjähriger Ablehnung Anerkennung fanden - wäre Kreativität ein leistungsintrinsisches Merkmal, wäre diese späte Anerkennung kaum $\mathrm{zu}$ erklären. Csikszentmihalyis Ansatz steht jedoch im Widerspruch zur traditionellen Ansicht, dass Kreativität die Schöpfung eines einzelnen genialen Geistes ist [86].

Der heute wohl am weitesten verbreiteten Definition zufolge ist Kreativität 
gleichbedeutend mit der Fähigkeit, etwas sowohl Neues als auch Wertvolles oder zumindest Angemessenes zu erschaffen [93]. Eine kreative Leistung muss danach nicht nur originell, sondern auch nützlich sein, um als solche erkannt zu werden. Für den Zusammenhang zwischen Kreativität und Schlaf sind jedoch vor allem die Ebenen kognitiver und neurobiologischer Prozesse relevant, die kreativen Leistungen in diesem Sinne unterliegen.

\section{Kognitionspsychologie der Kreativität}

In psychologischen Ansätzen sind die Modelle von Helmholtz [46] und Wallas [100] noch immer wirkmächtig, nach denen der kreative Prozess unterscheidbare Phasen beinhaltet, die z. T. auch von längerer Dauer sein können. $\mathrm{Zu} \mathrm{Be-}$ ginn steht hierbei die Vorbereitungspha$s e$, in der das nötige Handwerkszeug für das jeweilige Gebiet erlangt werden muss. Dieses Wissen ist eine Voraussetzung für die Identifikation und kreative Bearbeitung eines Problems. Da dieses jedoch meist nicht gleich gelöst werden kann, schließt sich daran eine Inkubationsphase an, in der nicht weiter explizit über einen Lösungsversuch nachgedacht wird, jedoch möglicherweise auf unbewusster Ebene problemrelevante kognitive Prozesse weiterarbeiten. In der Illuminationsphase kommt es dann zu einer schlagartigen Einsicht, in der eine Erklärung für die anfangs gestellte Fragestellung ins Bewusstsein dringt. In der sich daran anschließenden Verifikationsphase kommt es zu einer Bewertung der neu gewonnen Einsicht und zur endgültigen Ausarbeitung.

Die meisten psychologischen Modelle der Kreativität nehmen implizit oder explizit auf das Phasenmodell Bezug. So ging Kris [55] bspw. auf Grundlage psychodynamischer Theorien davon aus, dass neues Gedankengut durch ein primärprozesshaftes Eindringen ungeordneter Eingebungen ins Bewusstsein entspringe, das dann in einem zweiten Schritt sekundärprozesshaft ausgearbeitet werden könne. Eine weitere Möglichkeit stellte Campbell [11] nach dem Vorbild Darwins vor, indem er darauf hinwies, dass neues Wissen durch blinde Variation verschiedener Prozesse mit anschließender Selektion brauchbarer Ideen entstehen kann. Einem ähnlichen Gedanken folgend hatte Osborn [73] die Technik des Brainstormings entwickelt, bei der die Lösung eines Problems mithilfe der Generierung möglichst vieler unterschiedlicher Ideen in einer förderlichen Atmosphäre erfolgt, die erst in einem nachfolgenden Schritt bewertet werden. Mednick [68] postulierte flache Assoziationshierarchien als wesentliche Voraussetzung für kreative Leistungen: Eine Verknüpfung schwach miteinander assoziierter, semantisch weit auseinanderliegender kognitiver Elemente erlaubt nichtprotoypische und damit originelle Problemlösungen. Ähnlich hielt Mendelsohn [70] defokussierte

\section{Hier steht eine Anzeige}

\section{Springer}


Aufmerksamkeitsprozesse für kreativitätsförderlich, da durch die gleichzeitige Aktivierung mehrerer kognitiver Elemente die Wahrscheinlichkeit neuartiger problemrelevanter Assoziationen erhöht wird.

Im Gegensatz zur Annahme kreativitätsspezifischer kognitiver Prozesse postulierte Weisberg [101], dass nichts weiter als gewöhnliche kognitive Prozesse an kreativen Neuschöpfungen beteiligt seien und dass Kreativität u. a. lediglich eine Folge erworbenen Wissens darstelle [102]. Auch die Forgetting-Fixation-Hypothese von Smith und Blankenship [90] verzichtet auf kreativitätsspezifische kognitive Mechanismen mit dem Hinweis, dass eine Inkubationsphase eine Abkehr von der Beschäftigung mit einem unlösbaren Problem erlaubt, und so durch das Vergessen falscher Lösungswege rein passiv die Chance erhöht wird, durch alternative Wege zur Problemlösung zu gelangen. Ähnlich wurde auf die Rolle schlichten Zufalls als wesentlicher Treiber kreativer Prozesse hingewiesen, da dieser kognitive Elemente in chaotischer Weise aktiviert und miteinander assoziiert und dadurch von Zeit zu Zeit Zufallstreffer landet ([50, 51]; vgl. [11]).

Dem Geneplore-Modell von Finke et al. [34] zufolge besteht Kreativität aus einem generativen Teil, in dem potenziell gewinnbringende Ideen zusammengestellt werden, und einem explorativen bzw. evaluativen Teil, in dem das Rohmaterial untersucht, bewertet und verarbeitet wird. Das Hauptaugenmerk hierbei ist auf die mentalen Aspekte kreativer Prozesse gerichtet, etwa das Erleben plötzlicher Einsicht. Dabei kommt es infolge von unbewussten Prozessen zu einer schlagartigen Erkenntnis der richtigen Lösung für ein bestimmtes Problem [83]. Das Geneplore-Modell weist damit Gemeinsamkeiten mit dem traditionellen Phasenmodell von Helmholtz [46] und Wallas [100] auf, indem es zyklisch wiederholende Phasen verschiedener kreativer Prozesse annimmt.

Einige der Widersprüche zwischen verschiedenen theoretischen Konzepten und Modellen von Kreativität lassen sich auflösen, wenn statt einer einzelnen, starren Definition ein offener Kreativitätsbegriff angenommen wird, der sich in
Somnologie $2016 \cdot 20: 8-15 \quad$ DOI 10.1007/s11818-015-0039-z

(c) The Author(s) 2016

\section{Marisch · L. Genzel · A. Steiger · M. Dresler}

\section{Kreativität und Schlaf}

\section{Zusammenfassung}

Kreativität gilt als eine der höchstgeschätzten kognitiven Eigenschaften und Motor der menschlichen Kultur. Über die psychologischen und neurobiologischen Grundlagen kreativer Leistungen ist noch immer vergleichsweise wenig bekannt - u. a. aufgrund der Tatsache, dass Originalität und Einzigartigkeit als essenzielle Merkmale von Kreativität sich einer systematischen Untersuchung zu entziehen scheinen. Historische Anekdoten, theoretische Überlegungen und vermehrt auch empirische Studien deuten darauf hin, dass auch Schlaf eine Rolle in der Entstehung kreativer Leistungen spielen könnte: Schlaf konsolidiert nicht nur neu erworbene Informationen, sondern reorganisiert auch bestehende Wissensstrukturen und fungiert damit als Inkubationsphase kreativer Ideen und origineller Problemlösungen. Kognitive und physiologische Modelle betrachten Zufallsprozesse, eine Abkehr

\section{Creativity and Sleep}

\section{Abstract}

Creativity is one of the most valued cognitive traits and the driver of human culture. However, little is known about its psychological and neurobiological basis partly because originality and uniqueness as essential characteristics of creativity are elusive topics when it comes to systematic research. Historical anecdotes, theoretical considerations and an increasing number of empirical studies suggest that sleep plays a role in the genesis of creative achievements: sleep does not only help to consolidate newly acquired information, but also to reorganize existing knowledge structures, and hence functions as an incubation phase for creative ideas and original problem solutions. Cognitive and physiological models consider random processes, a renunciation from von erfolglosen Problemlösungsversuchen, flache Assoziationshierarchien, defokussierte Aufmerksamkeit oder eine schwache bzw. fluktuierende präfrontalkortikale Aktivierung als wesentliche Mechanismen kreativer Leistungen. Es fällt auf, dass diese Merkmale auch im Schlaf, insbesondere im REMSchlaf und in der Abfolge verschiedener Schlafstadien und Wachphasen über die nächtlichen Schlafzyklen hinweg, realisiert sind. Die systematische Erforschung der mit kreativer Inkubation verbundenen Reorganisation von Wissensstrukturen im Schlaf stellt mithin ein vielversprechendes und bislang zu wenig erforschtes Feld der kognitiven Neurowissenschaften dar.

Schlüsselwörter

Kreativität · Einsicht · Inkubation · REM . Gedächtnis unsuccessful problem solving attempts, flat associative hierarchies, defocused attention or low/fluctuating prefrontal cortical activity as essential mechanisms underlying creative achievements. Strikingly, these features are also realized during sleep, particularly during REM sleep and during the course of the nocturnal cycle of sleep stages. Systematic research on creative incubation processes which are realized in the reorganization of knowledge structures during sleep are a promising yet understudied field in cognitive neuroscience.

\section{Keywords}

Creativity · Insight · Incubation · REM . Memory
Abhängigkeit der Forschungsfrage und dazu passender Operationalisierung auf verschiedene Weise konkretisieren lässt [25]. Statt einer klar definierten Kreativität kann so auch von verschiedenen Kreativitäten gesprochen werden [92]. Paradigmatische Beispiele für solch heuristisch unterscheidbare Kreativitätskonzepte sind die bereits angesprochenen
Konzepte origineller Produktivität vs. kreativer Problemeinsicht [25]. Im Folgenden sollen beide Paradigmen der Kreativität betrachtet werden.

\section{Neurobiologie der Kreativität}

Aktuelle neurobiologische Ansätze der Kreativitätsforschung bauen zumeist auf 
psychologischen und kognitionswissenschaftlichen Modellen auf. So vereinigte z. B. Martindale [63] das Konzept der flachen Assoziationshierarchien von Mednick [68] mit der Idee defokussierter Aufmerksamkeit von Mendelsohn [70] in seiner Low-Arousal-Hypothese, nach der eine schwache Aktivierung mehrerer unterschiedlicher Hirnregionen die Verknüpfung weit auseinanderliegender Assoziationen im Sinne divergenten Denkens ermöglicht. Dies führte er unter anderem auf seine Beobachtungen zurück, dass hoch kreative Probanden bei der Ausführung von Kreativitätsaufgaben durch eine vergleichsweise geringe kortikale Aktivität charakterisiert waren [65]. Martindale und Hasenfus [64] replizierten diese Ergebnisse durch den Nachweis, dass bei kreativeren Teilnehmern während einer Inspirationsphase, in der sie sich eine Geschichte ausdenken sollten, eine geringere und variablere kortikale Aktivierung verzeichnet wurde als in der Ausarbeitungsphase, in der die Gedanken niedergeschrieben wurden. Dies konnte jedoch nur beobachtet werden, wenn die Probanden dazu angehalten wurden, bei ihrem Vorgehen so originell wie möglich zu sein. Unterstützung erfährt die Hypothese weiterhin durch eine Studie von Mölle et al. [71], in der während der Bearbeitung von divergenten Denkaufgaben Kreativitäts- mit EEG-Komplexitätsmaßen korrelierten. Die Ergebnisse wurden als stärkere funktionale Konnektivität zwischen unterschiedlichen Hirnarealen bei kreativeren Probanden interpretiert. Zahlreiche Studien zeigen eine erhöhte EEG-Alpha-Aktivität während kreativen Denkens, was als internal orientierter Aufmerksamkeitsprozess interpretiert wurde [33]. In diesem Zusammenhang wurde kürzlich auf die Bedeutung des Default Mode Network hingewiesen [4].

Neurochemisch wird neben Dopamin, das mit dem Antrieb zu kreativer Leistung in Verbindung gebracht wird [35], v. a. eine Rolle von Noradrenalin diskutiert [44]. So berichteten Kurup und Kurup [56], dass Schriftsteller und Dichter verringerte Noradrenalinspiegel aufwiesen. Darüber hinaus konnten Beversdorf et al. [6, 7] zeigen, dass Probanden nach der Einnahme des
$\beta$-Adrenozeptorantagonisten Propranolol besser beim Lösen von Anagrammen abschnitten.

Basierend auf den Annahmen von Galin [36], nach denen die rechte Hirnhemisphäre in einer primärprozesshaften Weise arbeiten würde, berichtete Martindale et al. [66], dass kreative Probanden bei der Bearbeitung von Kreativitätstests eine höhere rechtslaterale EEG-Aktivität aufwiesen. Auch funktionelle Bildgebungsstudien zu künstlerischer Kreativität [54] oder zu Einsichtserlebnissen unterstützen die Hypothese der Hemisphärenasymmetrie kreativen Denkens [ 8,52 , 62, 105].

Dem präfrontalen Kortex wird in den meisten neurobiologischen Ansätzen zur Kreativität eine herausragende Rolle zugeschrieben [12, 18, 31, 44, 45]. So wird z. B. rechtsseitige präfrontale Aktivität mit semantischem divergenten Denken in Verbindung gebracht [49]. Zudem zeigt sich eine stärkere rechtsseitige präfrontale Aktivierung bei kreativen gegenüber weniger kreativen Probanden während der Bearbeitung von Kreativitätsaufgaben [12, 54]. Aber auch linksseitige präfrontale Aktivität wird mit kreativen Kognitionen in Verbindung gebracht $[5,32]$. Eine Untersuchung an Jazzmusikern konnte hingegen eine beidseitige Deaktivierung des lateralen präfrontalen Kortex während spontaner kreativer Improvisation nachweisen [60].

Dass sowohl hohe als auch niedrige präfrontale Aktivität mit Kreativität in Zusammenhang gebracht wird, wirkt nur auf den ersten Blick widersprüchlich: Kreative Prozesse bestehen typischerweise aus mehrere Phasen mit jeweils verschiedenen Mechanismus und Zielsetzungen [34, 46, 100]. Ein einzelner neurobiologischer Prozess wäre mithin gar nicht als neuronales Korrelat der Kreativität schlechthin plausibel [19, 20, 80]. Tatsächlich konnte kürzlich in einer funktionellen Bildgebungsstudie gezeigt werden, dass verschiedene Phasen der Kreativität mit unterschiedlichen präfrontalen Aktivierungsmustern einhergehen: verstärkte ventromediale präfrontale Aktivität während der Phase der Ideengeneration, hingegen verstärkte dorsolateral präfrontale Aktivität während der Phase der Ideenevaluierung
[53]. Auf Netzwerkebene wird dieser Antagonismus durch das Zusammenspiel zwischen Default Mode Network und Executive Control Network abgebildet [4]. Vermeintliche Widersprüche in neurobiologischen Daten zur Kreativität können somit bei näherer Betrachtung als Bestätigung des traditionellen Phasenmodells der Kreativität interpretiert werden.

\section{Kreativität und Schlaf}

Einige der angesehensten kreativen Leistungen der menschlichen Geistesgeschichte werden anekdotisch auf eine Entstehung im Schlaf bzw. Traum zurückgeführt. Als Beispiele seien hier die Entschlüsselung der Inschrift des Steins von Nebukadnezar durch Hilprecht, die Erfindung der Nähmaschine durch Howe, die Erstellung des Periodensystems durch Mendelejew, die Dichtung von „Kubla Kahn“ durch Coleridge oder die Komposition der „Teufelstrillersonate“ durch Tartini, genannt [3, 17, 23, 24, 67].

Eine Reihe systematischer Studien bestätigt diese anekdotische historische Evidenz. So konnten Wagner et al. [98] demonstrieren, dass doppelt so viele Testteilnehmer nach einem 8-stündigen Schlaf eine versteckte, abkürzende Regelhaftigkeit in einer gestellten Aufgabe entdeckten als die Kontrollgruppe, die nicht schlafen durfte, sodass die Autoren daraus folgerten, dass Schlafen das Erlangen von Einsicht in eine Problemlösung zu fördern scheint. Auch für die Übertragung einer bekannten Problemlösung auf ein neues, aber analoges, Problem hat sich Schlaf als förderlich erwiesen [72]. Sio et al. [88] fanden anhand eines Remote Associate Task Hinweise, dass der Schlaf v. a. für die Lösung schwieriger Fragestellungen hilfreich sei. Dies stimmt mit einer neueren Studie der Autoren überein, nach der einfache Probleme durch fokussierte und schwierige Probleme durch defokussierte Aufmerksamkeit profitieren [89]. Dass tatsächlich aktive Inkubationsprozesse im Schlaf kreativitätsfördernd wirken, konnten Ritter et al. [78] zeigen: Wurden Probanden bei der Bearbeitung der Versuchsaufgaben einem Duft ausgesetzt und dieser auch anschließend 
im Schlafen appliziert, waren sie nicht nur kreativer in ihren Lösungsansätzen der vor dem Schlafen gestellten Aufgabe, sondern auch besser darin, ihre kreativste Idee zu selektieren. Die Autoren folgerten daraus, dass die mit dem Duft assoziierte Aufgabenstellung im Schlaf reaktiviert und dadurch potenzielle Problemlösungen inkubiert wurden. Aber auch unabhängig von Inkubationsprozessen fördert Schlaf Kreativität insofern, als nach einer Nacht Schlafentzug Flexibilität und Originalität bei Kreativitätsaufgaben beeinträchtigt sind [48].

\section{Kreativität und Schlaf-EEG}

Einige Studien haben versucht, Kreativität mit spezifischen Schlafstadien oder EEG-Mikroprozessen in Verbindung zu bringen. Cai et al. [10] zeigten in einer Mittagsschlafstudie, dass insbesondere REM-Schlaf - im Vergleich zu NREMSchlaf oder Wachphasen - kreativitätsfördernd wirkt, sofern entsprechende Aufgaben vor dem Schlaf gestellt und nach dem Schlaf bearbeitet wurden. Nach Weckungen aus dem REM-Schlaf im Vergleich zu NREM-Weckungen schnitten Probanden besser beim Lösen von Anagrammen ab [99] und waren $\mathrm{zu}$ weitreichenderen Assoziationen fähig [91]. Die Unterdrückung von REMSchlaf wirkte sich zudem negativ auf die Produktion kreativer Antworten aus [41, 58]. Darüber hinaus führen Aufgaben, die divergentes Denken anregen, zu einem erhöhten Anteil an REM-Schlaf im folgenden Nachtschlaf [57].

Yordanova et al. [104] hingegen identifizierten EEG-Alphaaktivität während des Tiefschlafs als Marker für den schlafinduzierten Übergang von implizitem Wissen in explizite Einsicht. Dabei war ebenfalls eine erhöhte $\beta$-Aktivität im Tiefschlaf zu beobachten, wenn die Probanden nach dem Schlafen Einsicht erlangten, unabhängig davon, was sich vor dem Schlafen ereignete [105]. Auch Drago et al. [22] fanden Zusammenhänge wischen Kreativität und NREMSchlaf, konkret mit zyklisch alternierenden Arousalmustern (CAP). So bestand eine positive Korrelation zwischen CAP, v. a. des Subtyps A1, und der Originali- tät der Probanden. Die Generation des Subtyps A1 wird v. a. im Frontalkortex verortet [30]. REM-Schlaf hingegen korrelierte in dieser Studie negativ mit der Originalität. Weiterhin wies sowohl das Schlafstadium 1 mit der Flexibilität und Ideenflüssigkeit, als auch das Schlafstadium 4 mit der Originalität und der figuralen Kreativität eine positive Korrelation auf [22].

Die wenigen Studien zum Zusammenhang zwischen Kreativität und Elektrophysiologie des Schlafs kommen mithin zu uneinheitlichen Ergebnissen - zwar können REM-Schlafprozesse als theoretisch und empirisch naheliegendste Mechanismen einer kreativitätsförderlichen Wirkung des Schlafs betrachtet werden, gegenteilige Befunde und kreativitätsförderliche Aspekte des NREMSchlafs machen die Situation jedoch unübersichtlich. Welche Schlafstadien und Schlaf-EEG-Mikroprozesse in welcher Weise welche kreativen Prozesse fördern, wird eine der wesentlichen Aufgaben zukünftiger Forschung in diesem Bereich sein. Auf derzeitigem Forschungsstand kann nur spekuliert werden, dass Prozesse der Gedächtnisintegration und -reorganisation im Schlaf eine wesentliche Rolle dabei spielen werden. Aktuelle Entwicklungen im Bereich der Forschung zu Gedächtnisschemata könnten hierbei neue Forschungswege eröffnen: Als Gedächtnisschemata werden vorhandene Wissensstrukturen verstanden, in die neue, jedoch semantisch verwandte Informationen integriert werden und dadurch schneller erlernt und konsolidiert werden können. Kreative Einsichten können in diesem Zusammenhang als Erkenntnis bzw. Vervollständigung eines problemrelevanten Schemas verstanden werden. Neurobiologisch gelten Gedächtnisschemata als wesentlich durch den ventromedialen Präfrontalkortex vermittelt [97], also einer Hirnstruktur, die sowohl während der Ideengeneration [53] als auch im REM-Schlaf [9] starke Aktivierung aufweist. Es wird weiterhin diskutiert, inwiefern Gedächtnisschemata im Schlaf aufgebaut werden [59] und ihre konsolidierungsfördernde Wirkung insbesondere im REM-Schlaf vermitteln [29].

\section{Kreativität und Traum}

Neben der physiologischen Ebene bestätigen auch zahlreiche Studien zum Träumen einen Zusammenhang zwischen Schlaf und Kreativität. Phänomenologisch sind Träume durch kognitive Ablenkbarkeit, gelockerte Assoziationen und eine ungewöhnliche, häufig bizarre, Folge von Bildern und Handlungen gekennzeichnet und weisen somit die in kognitiven Modellen beschriebenen Kreativitätsvoraussetzungen auf: Flache Assoziationshierarchien [68] und defokussierte Aufmerksamkeit [70], die $\mathrm{zu}$ originellen Verbindungen weit auseinanderliegender kognitiver Elemente führen. Um die Frage zu klären, inwieweit Träume das kreative Leben von Menschen beeinflussen, führten Schredl und Erlacher [85] eine Befragung von 1080 Teilnehmern durch. $8 \%$ aller Träume wirkten sich danach auf die Kreativität der Betreffenden aus, wobei Probanden mit größerem Interesse am Gebrauch traumhafter Elemente auch eine größere alltägliche Beeinflussung durch Träume aufwiesen [74]. Kreative Individuen können sich nicht nur häufiger an ihre Träume erinnern [84], ihre Träume sind auch ideenreicher [82], einzigartiger und komplexer [96] sowie vermehrt von primärprozesshaftem Denken geprägt [21, 40]. Kritisiert wurden Berichte über den Zusammenhang zwischen Trauminhalten und Kreativität jedoch mit dem Hinweis auf verschiedene Traumlängen, die möglicherweise Artefakte in den Befunden erzeugen $[61,81,101]$.

Um Träume aktiv für kreative Zwecke einsetzen zu können, existieren zahlreiche Ratschläge, wie das Traumgeschehen aktiv moduliert und genutzt werden kann $[16,37]$. So empfahl Delaney [16] bspw. einen Traum zu inkubieren, indem man sich bewusst mit einer Frage, deren Beantwortung man wünscht, schlafen legt, um Anregungen diesbezüglich zu erhalten. In einem direkten Vergleich dieser Technik mit einer Entspannungsübung konnten White und Taytroe [103] aufzeigen, dass die Teilnehmer sich weniger gestresst fühlten und bezüglich der Problemlösung Fortschritte machten. Dabei scheinen Probleme persönlicher Natur eher einer Bewältigung zugänglich zu 
sein als akademische oder generelle Herausforderungen [2]. Auch hypnotisch induzierte Träume zur Überwindung kreativer Blockaden wurden als kreativitätsförderlich berichtet [15]. Besonders effektiv könnten Ansätze sein, luzide Träume zur kreativen Inkubation einzusetzen: Die Verbindung aus gelockerter Assoziativität des Traumzustands mit erhaltender kognitiver Kontrolle und Kritikfähigkeit erlaubt neben einer gezielten Ideengenerierung auch eine umgehende Ideenbewertung während des luziden Traums [95].

\section{Fazit}

Verschiedene empirische Studien bestätigen eine weitgehend anekdotische historische Evidenz, nach der Schlafkreative Leistungen unterstützt. Bereits das klassische Phasenmodell von Wallas [100] und Helmholtz [46] beschrieb eine Inkubationsphase als wesentlichen Teil kreativer Arbeit. Die kreativitätsfördernde Wirkung des Schlafs in diesem Sinne kann dabei auf unterschiedliche Weise gedeutet werden: Eher passiv orientierte Ansätze betonen die radikale Abkehr von der bewussten Beschäftigung mit einem unlösbaren Problem im Schlaf, wodurch falsche Lösungswege aus dem Aufmerksamkeitsfokus rücken und so das Verfolgen alternativer Lösungsversuche ermöglicht wird. Andere Ansätze hingegen gehen von aktiven kognitiven Prozessen im Schlaf aus, die unbewusst arbeiten und deren Ergebnis als plötzliche Einsicht zu Tage tritt [94]. Dazwischen liegen Ansätze, die auf flache Assoziationshierarchien, defokussierte Aufmerksamkeit, und Zufallsprozesse verweisen, nach denen Schlaf und Traum semantisch weit auseinanderliegende kognitive Elemente in chaotischer Weise aktivieren und miteinander assoziieren und dadurch von Zeit zu Zeit problemrelevante ,Zufallstreffer' landen ([11, 50, 51, 68, 70]).

Nach neurobiologischen Ansätzen wie der Low-Arousal-Hypothese [63] entsteht Kreativität in einem Zustand unfokussierter Aufmerksamkeit, etwa aufgrund verringerter präfrontaler Aktivierung und noradrenerger Demodulation. Dies ermöglicht während des Schaffensprozesses eine gleichzeitige
Repräsentation vieler unterschiedlicher Assoziationen, die dann zu kreativen Kognitionen neu verknüpft werden können. Ideale Voraussetzungen dafür bietet im Leicht- und Tiefschlaf ein niedriges kortikales Arousal und im REM-Schlaf neben einer niedrigen noradrenergen Transmission v. a. die chaotische Aktivierung des Kortex durch den Hirnstamm und die fehlende Inhibierung und Kontrolle der dadurch aktivierten Kognitionen durch die niedrige Aktivität des präfrontalen Kortex [47]. Aufgrund des fehlenden stetigen sensorischen Inputs durch die Außenwelt können die REM-Schlaf-bedingten Zufallsaktivierungen, relativ aktuelle Erlebnisse und Probleme sowie ältere Gedächtnisinhalte ungestört miteinander verglichen und integriert werden $[42,75]$, wodurch die Wahrscheinlichkeit einer kreativen Kombination von neuen Ideen mit problemrelevantem Vorwissen steigt. Die Kombination aus deaktiviertem dorsolateralen und aktiviertem ventromedialen präfrontalen Kortex während des REMSchlafs [9] erlaubt dabei eine disinhibierte Ideengeneration [53, 60]. Zum Bewusstwerden und Bewerten solcher kreativer Kombinationen ist dann jedoch wieder die Aktivierung des präfrontalen Kortex notwendig [53] - wofür die zahlreichen kurzen Wachphasen, insbesondere beim Schlafphasenwechsel, eine Möglichkeit bieten, was im Falle luzider Träume jedoch bereits im Schlaf möglich sein könnte [26, 28, 95].

Die alltäglich wiederkehrenden $\mathrm{Zu}$ stände des Schlafens und Träumens bieten mithin ein ideales neurobiologisches Milieu, um die Funktion einer erfolgreichen Inkubationsphase kreativer Ideen und Leistungen zu erfüllen. Die hier beschriebenen neurobiologischen Zusammenhänge zwischen Kreativität und Schlaf sind intuitiv plausibel, beruhen jedoch größtenteils auf jeweils eigenständigen Studien innerhalb der Kreativitäts- und Schlafforschung. Ihre Verbindung ist mithin noch weitgehend spekulativ. Die kognitive Neurowissenschaft des Schlafs hat in den vergangenen Jahren weitreichende und faszinierende Fortschritte in der Erforschung der neuronalen Mechanismen der Konsolidierung und Integration neuer Informa-
Hier steht eine Anzeige.

Springer 
tionen gemacht $[38,76]$. Nicht zuletzt legt die wesentliche Bedeutung kreativer Leistungen für die Wissenschaft und für moderne Wissensgesellschaften im Allgemeinen nahe, dass eine systematische Erforschung der, mit kreativer Inkubation verbundenen, Reorganisation von Wissensstrukturen im Schlaf sich zu einem ähnlich vielversprechenden Feld entwickeln könnte.

\section{Korrespondenzadresse}

\section{Dr. Martin Dresler}

Donders Institute, Radboud University Medical Centre

Kapittelweg 29, 6525 EN Nijmegen,

Niederlande

martin.dresler@donders.ru.nl

Danksagung. Open access funding provided by Max Planck Society (or associated institution if applicable). Die vorliegende Arbeit entstand im Rahmen einer Förderung durch die VolkswagenStiftung. Teile dieses Beitrags bauen auf der Dissertation von Cynthia Marisch an der medizinischen Fakultät der LudwigMaximilians-Universität München auf.

\section{Einhaltung ethischer Richtlinien}

Interessenkonflikt. C. Marisch, L. Genzel, A. Steiger und M. Dresler geben an, dass kein Interessenkonflikt besteht.

Dieser Beitrag beinhaltet keine Studien an Menschen oder Tieren.

Open Access. This article is distributed under the terms of the Creative Commons Attribution 4.0 International License (http://creativecommons.org/ licenses/by/4.0/), which permits unrestricted use, distribution, and reproduction in any medium, provided you give appropriate credit to the original author(s) and the source, provide a link to the Creative Commons license, and indicate if changes were made.

\section{Literatur}

1. Abraham A, Windmann S (2007) Creative cognition: the diverse operations and the prospect of applying a cognitive neuroscience perspective. Methods 42:38-48

2. Barrett D (1993) The committee of sleep: a study of dream incubation for problem solving. Dreaming 3(2):115-122

3. Barrett D (2001) The committee of sleep: how artists, scientists, and athletes use dreams for creative problem-solving - and how you can too, 1. Aufl. Crown Business, New York

4. Beaty RE, Benedek M, Silvia PJ, Schacter DL (2015) Creative cognition and brain network dynamics. Trends Cogn Sci:doi:10.1016/j.tics.2015.10.004

5. Benedek M, Jauk E, Fink A, Koschutnig K, Reishofer G, Ebner F, Neubauer AC (2013) To create or to recall? Neural mechanisms underlying the generation of creative new ideas. Neuroimage 88C:125-133

6. Beversdorf DQ, Hughes JD, Steinberg BA, Lewis LD, Heilman KM (1999) Noradrenergic modulation of cognitive flexibility in problem solving. Neuroreport 10(13):2763-2767

7. Beversdorf DQ, White DM, Chever DC, Hughes JD, Bornstein RA (2002) Central ß-adrenergic modulation of cognitive flexibility. Neuroreport 13(18):2505-2507

8. Bowden EM, Jung-Beeman M (2003) Aha! Insight experience correlates with solution activation in the right hemisphere. Psychon Bull Rev 10(3):730-737

9. Braun AR, Balkin TJ, Wesensten NJ, Gwadry F, Carson RE, Varga M, Baldwin P, Belenky G Herscovitch P (1998) Dissociated pattern of activity in visual cortices and their projections during human rapid eye movement sleep. Science 279(5347):91-95

10. Cai DJ, Mednick SA, Harrison EM, Kanady JC, Mednick SC (2009) REM, not incubation, improves creativity by priming associative networks. Proc Natl Acad Sci USA 106(25):10130-10134

11. Campbell DT (1960) Blind variation and selective retention in creative thought as in other knowledge processes. Psychol Rev 67(6):380-400

12. Carlsson I, Wendt PE, Risberg J (2000) On the neurobiology of creativity. Differences in frontal activity between high and low creative subjects. Neuropsychologia 38(6):873-885

13. Csikszentmihalyi M (1997) Kreativität: Wie Sie das Unmögliche schaffen und Ihre Grenzen überwinden, 2. Aufl. Klett-Cotta, Stuttgart

14. Csikszentmihalyi M (1999) Implications of a systems perspective for the study of creativity. In: Sternberg RJ (Hrsg) Handbook of creativity. Cambridge University Press, Cambridge, S313-335

15. Davé R (1979) Effects of hypnotically induced dreams on creative problem solving. J Abnorm Psychol 88(3):293-302

16. Delaney G (1988) Lebe Deine Träume: Anleitung zum aktiven Träumen. mvg, München

17. Dement WC (1978) Some must watch while some must sleep: Exploring the world of sleep. W.W. Norton \& Co., New York

18. Dietrich A (2004) The cognitive neuroscience of creativity. Psychon Bull Rev 11(6):1011-1026

19. Dietrich A (2007) Who's afraid of a cognitive neuroscience of creativity? Methods 42(1):22-27

20. Dietrich A, Kanso R (2010) A review of EEG, ERP, and neuroimaging studies of creativity and insight. Psychol Bull 136(5):822-848

21. Domino G (1976) Primary process thinking in dream reports as related to creative achievement. J Consult Clin Psychol 44(6):929-932

22. Drago V, Foster PS, Heilman KM, Aricò D, Williamson J, Montagna P, Ferri R (2011) Cyclic alternating pattern in sleep and its relationship to creativity. Sleep Med 12(4):361-366

23. Dreistadt R (1971) An analysis of how dreams are used in creative behavior. Psychology 8(1):24-50

24. Dresler M (2008a) Kreativität und Schlaf. In: Dresler M, Baudson TG (Hrsg) Kreativität. Hirzel, Stuttgart

25. Dresler M (2008b) Kreativität als offenes Konzept In: Dresler M, Baudson TG (Hrsg) Kreativität. Hirzel, Stuttgart

26. Dresler M, Wehrle R, Spoormaker VI, Koch SP, Holsboer F, Steiger A, Obrig H, Sämann PG, Czisch M (2012) Neural correlates of dream lucidity obtained from contrasting lucid versus non-lucid REM sleep: a combined EEG/fMRI case study. Sleep 35(7):1017-1020
27. Dresler $M$, Spoormaker VI, Beitinger $P$, Czisch $M$, Kimura M, Steiger A, Holsboer F (2014) Neuroscience-driven discovery and development of sleep therapeutics. Pharmacol Ther 141(3):300-334

28. Dresler M, Erlacher D, Czisch M, Spoormaker VI (2016) Lucid dreaming. In: Kryger M, Roth T, Dement W (Hrsg) Principles and Practice of Sleep Medicine. Elsevier, Amsterdam (im Druck)

29. Durrant SJ, Cairney SA, McDermott C, Lewis PA (2015) Schema-conformant memories are preferentially consolidated during REM sleep. Neurobiol Learn Mem 122:41-50

30. Ferri R, Bruni O, Miano S, Terzano MG (2005) Topographic mapping of the spectral components of the cyclic alternating pattern (CAP). Sleep Med 6(1):29-36

31. Fink $A$, Grabner $R H$, Benedek $M$, Neubauer $A C$ (2006) Divergent thinking training is related to frontal electroencephalogram alpha synchronization. Eur J Neurosci 23(8):2241-2246

32. Fink A, Grabner RH, Benedek M, Reishofer G, Hauswirth V, Fally M, Neuper C, Ebner F, Neubauer AC (2009) The creative brain: investigation of brain activity during creative problem solving by means ofEEG and FMRI. Hum Brain Mapp 30(3):734-748

33. Fink A, Benedek M (2014) EEG alpha power and creative ideation. Neurosci Biobehav Rev 44:111-123

34. Finke RA, Ward TB, Smith SM (1992) Creative cognition: Theory, research, and applications. MIT Press, Cambridge, MA

35. Flaherty AW (2005) Frontotemporal and dopaminergic control of idea generation and creative drive. JComp Neurol 493(1):147-153

36. Galin D (1974) Implications forpsychiatry of leftand right cerebral specialization: A neurophysiological context for unconscious processes. Arch Gen Psychiatry 31(4):572-583

37. GarfieldP (1986) Kreativ träumen. Knaur, München

38. Genzel L, Kroes MC, Dresler M, Battaglia FP (2014) Light sleep versus slow wave sleep in memory consolidation: a question of global versus local processes? Trends Neurosci 37(1):10-19

39. Genzel L, Spoormaker VI, Konrad BN, Dresler M (2015) The role of rapid eye movement sleep for amygdala-related memory processing. Neurobiol Learn Mem 122:110-121

40. Giovacchini PL (1966) Dreams and the creative process. Br J Med Psychol 39(2):105-115

41. Glaubman H, Orbach I, Aviram O, Frieder I, Frieman M, Pelled O, Glaubman R (1978) REM deprivation and divergent thinking. Psychophysiology 15(1):75-79

42. Greenberg R (1987) The dream problem and problems in dreams. In: Glucksman ML, Warner $\mathrm{SL}$ (Hrsg) Dreams in new perspective. Human Sciences Press, New York

43. Guilford JP (1950)Creativity. AmPsychol5:444-454

44. Heilman KM (2005) Creativity and the Brain. Psychology Press, New York

45. Heilman KM, Nadeau SE, Beversdorf DO (2003) Creative innovation: possible brain mechanisms. Neurocase 9(5):369-379

46. von Helmholtz H (1896) Vorträge und Reden. Vieweg, Braunschweig

47. Hobson JA, Pace-Schott EF (2002) The cognitive neuroscience of sleep: neuronal systems, consciousness and learning. Nat Rev Neurosci 3(9):679-693

48. Horne JA (1988) Sleeploss and "divergent" thinking ability. Sleep 11(6):528-536

49. Howard-Jones PA, Blakemore SJ, Samuel EA, Summers IR, Claxton G (2005) Semantic divergence and 
creative story generation: an fMRI investigation. Cogn Brain Res 25(1):240-250

50. Jones RM (1987) Dreams and creativity. In: Russo RA (Hrsg) Dreams are wiser than men. North Atlantic Books, Berkeley

51. Jones RM (1999) Dream reflection and creative writing. In: Ullman M, Limmer C (Hrsg) The variety of dream. Experience, Albany

52. Jung-Beeman $M$, Bowden EM, Haberman J, Frymiare JL, Arambel-Liu S, Greenblatt R, Reber PJ, Kounios J (2004) Neural activity when people solve verbal problems with insight. PLoS Biol 2(4):500-510

53. Kleinmitz OM, Abecassis D, Shamay-Tsoory SG (2015) The neural underpinning of creativity and musical expertise Poster at the $1^{\text {st }}$ International Convention of Psychological Science, Amsterdam, 12-14 March

54. Kowatari Y, Lee SH, Yamamura H, Nagamori Y, Levy P, Yamane S, Yamamoto M (2009) Neural networks involved in artistic creativity. Hum Brain Mapp 30(5):1678-1690

55. Kris E (1965) Psychoanalytic explorations in art, 3. Aufl. International Universities Press, New York

56. Kurup RK, Kurup PA (2003) Hypothalamic digoxin, cerebral chemical dominance and myalgic encephalomyelitis. Int J Neurosci 113(5):683-701

57. Lewin I, Gombosh D (1973) Increase in REM time as a function of the need for divergent thinking. In: Koella WP, Levin P(Hrsg) Sleep. Karger, Basel

58. Lewin I, Glaubman H (1975) The effect of REM deprivation: is it detrimental, beneficial, or neutral? Psychophysiology 12(3):349-353

59. Lewis PA, Durrant SJ (2011) Overlapping memory replay during sleep builds cognitive schemata. Trends Cogn Sci 15(8):343-351

60. Limb CJ, Braun AR (2008) Neural substrates of spontaneous musical performance: an FMRI study of jazz improvisation. PLoSOne 3(2):e1679

61. Livingston G, Levin R (1991) The effects of dream length on the relationship between primary process in dreams and creativity. Dreaming 1(4):301-309

62. Luo J, Niki K (2003) Function of hippocampus in "insight" of problem solving. Hippocampus 13(3):316-323

63. Martindale C (1999) Biological Bases of Creativity. In: Sternberg RJ (Hrsg) Handbook of creativity. CambridgeUniversity Press, Cambridge, S137-152

64. Martindale C, Hasenfus N (1978) EEG differences as a function of creativity, stage of the creative process, and effort to be original. Biol Psychol 6(3):157-167

65. Martindale C, Hines D (1975) Creativity and cortical activation during creative, intellectual and EEG feedback tasks. Biol Psychol 3(2):91-100

66. Martindale C, Hines D, Mitchell L, Covello E (1984) EEG alpha asymmetry and creativity. Pers Individ Differ 5(1):77-86

67. Mazzarello P (2000) What dreams may come? Nature 408(6812):523

68. Mednick SA (1962) The associative basis of the creative process. Psychol Rev 69(3):220-232

69. Mednick SC, Cai DJ, Kanady J, Drummond SP (2008) Comparing the benefits of caffeine, naps and placebo on verbal, motor and perceptual memory. Behav Brain Res 193(1):79-86

70. Mendelsohn GA (1976) Associative and attentional processes in creative performance. J Pers 44(2):341-369

71. Mölle M, Marshall L, WolfB, Fehm HL, Born J (1999) EEG complexity and performance measures of creative thinking. Psychophysiology 36(1):95-104
72. Monaghan P, Sio UN, Lau SW, Woo HK, Linkenauge SA, Ormerod TC (2015) Sleep promotes analogical transfer in problem solving. Cognition 143:25-30

73. Osborn AF (1953) Applied Imagination: Principles and procedures of creative thinking. Scribner, New York

74. Pagel JF, Kwiatkowski CF (2003) Creativity and dreaming: Correlation of reported dream incorporation into waking behavior with level and type of creative interest. Creat Res J 15(2-3):199-205

75. Palombo SR (1987) Dreams: new frontiers. In: Glucksman ML, Warner SL (Hrsg) Dreams in new perspective. Human Sciences Press, New York

76. Rasch B, Born J (2013) About sleep's role in memory. Physiol Rev 93(2):681-766

77. Rhodes M (1961) An analysis of creativity. Phi Delta Kappan 42(7):305-310

78. Ritter SM, Strick M, Bos MW, Van Baaren RB, Dijksterhuis A (2012) Good morning creativity: task reactivation during sleep enhances beneficial effect of sleep on creative performance. J Sleep Res 21(6):643-647

79. Runco MA (2007) To understand is to create: an epistemological perspective on human nature and personal creativity. In: Richards R (Hrsg) Everyday creativity and new views of human nature: psychological, social, and spiritual perspectives. American Psychological Association, Washington DC, S91-107

80. Sandkühler S, Bhattacharya J (2008) Deconstructing insight: EEG correlates of insightful problem solving. PLoSOne 3(1):e1459

81. Saredi R, Baylor GW, Meier B, Strauch I (1997) Current concerns and REM-dreams: a laboratory study of dream incubation. Dreaming 7(3):195-208

82. Schechter N, Schmeidler GR, Staal M (1965) Dream reports and creative tendencies in students of the arts, sciences, and engineering. J Consult Psychol 29(5):415-421

83. Schooler JW, Melcher J (1995) The ineffability of insight. In: Smith SM, Ward TB, Finke RA (Hrsg) The creative cognition approach. MIT Press, Cambridge, Massachusetts

84. Schredl M (1995) Creativity and dream recall. J Creat Behav 29(1):16-24

85. Schredl M, Erlacher D (2007) Self-reported effects of dreams on waking-life creativity: an empirical study. J Psychol 141(1):35-46

86. Simonton DK (1999) Origins of genius: Darwinian perspectives on creativity. Oxford University Press, New York, NY

87. Simonton DK (2000) Cognitive, personal, developmental, and social aspects. Am Psychol 55(1):151-158

88. Sio UN, Monaghan P, Ormerod T (2013) Sleep on it but only if it is difficult: effects of sleep on problem solving. Mem Cogn 41(2):159-166

89. Sio UN, Ormerod TC (2015) Incubation and cueing effects in problem-solving: set aside the difficult problems but focus on the easy ones. Think Reason: doi:10.1080/13546783.2014.886626

90. Smith SM, Blankenship SE (1989) Incubation effects. Bull Psychon Soc 27(4):311-314

91. Spitzer M, Walder S, Clarenbach P (1993) Aktivierte assoziativeNetzwerkeimREM-Schlaf:semantische Bahnungseffekte nach dem Aufwecken aus verschiedenen Schlafstadien. In: Meier-Ewert K, Rüther E (Hrsg) Schlafmedizin. Gustav Fischer, Stuttgart, S168-178

92. Sternberg RJ (2005) Creativity or creativities? Int J Hum-Computer Stud 63(4):370-382

93. Sternberg RJ (2006) The nature of creativity. Creat ResJ 18(1):87-98
94. Stokes D (2007) Incubated cognition and creativity. JConscious Stud 14(3):83-100

95. Stumbrys T, Daniels M (2010) An exploratory study of creative problem solving in lucid dreams: preliminary findings and methodological considerations. Int JDream Res 3(2):121-129

96. Sylvia WH, Clark PM, Monroe LJ (1978) Dream reports of subjects high and low in creative ability. J Gen Psychol 99(2):205-211

97. van Kesteren MT, Rijpkema M, Ruiter DJ, Fernández G (2010) Retrieval of associative information congruent with prior knowledge is related to increased medial prefrontal activity and connectivity. J Neurosci 30:15888-15894

98. Wagner U, Gais S, Haider H, Verleger R, Born J (2004) Sleep inspires insight. Nature 427(6972):352-355

99. Walker MP, Liston C, Hobson JA, Stickgold R (2002) Cognitive flexibility across the sleep-wake cycle: REM-sleep enhancement of anagram problem solving. Cogn Brain Res 14(3):317-324

100. Wallas G (1926) The art of thought. Cape, London

101. Weisberg RW (1986) Creativity: genius and other myths. Freeman, New York

102. Weisberg RW (1999) Creativity and knowledge: a challenge to theories. In: Sternberg RJ (Hrsg) Handbook of creativity. Cambridge University Press, Cambridge, S226-250

103. White GL, Taytroe L (2003) Personal problemsolving using dream incubation: dreaming, relaxation, or waking cognition? Dreaming 13(4):193-209

104. Yordanova J, Kolev V, Wagner U, Born J, Verleger $\mathrm{R}$ (2012) Increased alpha (8-12 Hz) activity during slow wave sleep as a marker for the transition from implicit knowledge to explicit insight. J Cogn Neurosci 24(1):119-132

105. Yordanova J, Kolev V, Wagner U, Verleger R (2010) Differential associations of early-and late-night sleep with functional brain states promoting insight to abstract task regularity. PloS One 5(2):e9442 